\title{
Two-phase coexistence in Fe-Ni alloys synthesized by ball milling
}

\author{
L. B. Hong and B. Fultz \\ Division of Engineering and Applied Science, California Institute of Technology, 138-78, Pasadena, \\ California 91125
}

(Received 13 November 1995; accepted for publication 2 January 1996)

\begin{abstract}
We used mechanical alloying with a Spex 8000 mixer/mill to synthesize a series of $\mathrm{Fe}_{100-x} \mathrm{Ni}_{x}$ alloys from $x=0$ to $x=49$. The Spex mill was modified so that it could also operate at a reduced milling intensity, and we compared the alloys synthesized after long times with the normal and reduced milling intensities. X-ray diffractometry and Mössbauer spectrometry were used to measure the volume fractions of the bcc and fcc phases in the alloys, and to determine the chemical compositions of the individual phases. We found that the composition ranges of the bcc and fcc single phase regions were extended well beyond their equilibrium ranges. At the higher milling intensity, we found that the bcc phase was destabilized with respect to the fcc phase, and the two-phase region shifted to lower Ni concentrations. For those alloys with coexisting bcc and fcc phases, we present evidence that the chemical compositions of the two phases are nearly the same. We explain the destabilization of the bcc with milling intensity as originating with a higher defect density in the bcc alloys than in the fcc alloys. We argue that this defect density is not homogeneous throughout the alloy, however, and the distribution of defect enthalpies can explain the twophase coexistence in the as-milled alloys. () 1996 American Institute of Physics. [S0021-8979(96)01508-5]
\end{abstract}

\section{INTRODUCTION}

Although ball milling has been used for many years for the synthesis of dispersion-strengthened superalloys for jet engine parts, ${ }^{1}$ recently it has attracted much attention from the materials science community, in large part because of the discovery that ball milling can cause solid-state amorphization. ${ }^{2-16}$ Nanocrystalline materials with grain sizes of typically $10 \mathrm{~nm}$ are another class of materials of recent interest than can be synthesized by ball milling. ${ }^{13-16}$ Many types of phase transformations have been observed during ball milling, such as polymorphic transformations of compounds and disordering of ordered alloys. ${ }^{17-25}$ It is also wellestablished that high energy ball milling can be used to synthesize alloy phases with extended solid solubilities, ${ }^{13-15,26-40}$ for which the Hume-Rothery systematics for size and electronegativity may be relevant. ${ }^{37}$

The applicability of thermodynamics to materials synthesis by ball milling is a subject of ongoing investigation. It is generally expected that the synthesis of new materials by ball milling depends on the thermochemistry of the alloy, plus the microstructural defects generated during ball milling. Some interpretations of the steady-state phases formed during milling have used model free energy calculations (of the "CALPHAD" style) with polymorphic constraints to restrict processes of chemical segregation. ${ }^{41-47}$ The general preferences of $\mathrm{Fe}-\mathrm{Cu}$ alloys for the bcc or fcc structure can be understood in this way. ${ }^{41-44,47}$ For compositions from $20 \%-40 \% \mathrm{Cu}$, however, as-milled $\mathrm{Fe}-\mathrm{Cu}$ alloys have a two-phase microstructure comprising bcc and fcc phases. ${ }^{38-44,47,48}$ Such a two-phase coexistence cannot be predicted by a conventional free-energy argument with a polymorphic constraint. For a specific composition and temperature, only one phase can have the lowest free energy. Furthermore, there is evidence from x-ray lattice parameter measurements that the atomic level mixing induced by ball milling also serves to homogenize the alloy chemistry, so the bcc and fcc phases may have similar chemical compositions. ${ }^{38,39}$

For testing the validity of thermodynamic arguments it is useful to vary the chemical composition of the alloy, but control over the temperature ${ }^{49-52}$ and milling intensity ${ }^{53-57}$ may also be useful. In the present study, we synthesized $\mathrm{Fe}-\mathrm{Ni}$ alloys with a range of chemical compositions, and we milled them at two different intensities. Our interest was in understanding how milling intensity affected the region of two-phase (bcc plus fcc) coexistence that we found from about 15-34 at.\% Ni. Model free-energy calculations (with Thermo-Calc software) were performed for $\mathrm{Fe}-\mathrm{Ni}$ alloys, and the general preference of the alloys for fcc or bcc structures was predicted from the calculated polymorphic transformation composition (the Ni composition at the intersection of the bcc and fcc free-energy curves, which was $28 \%$ at low temperature). With increased milling intensity, the region of two phase coexistence was found to expand asymmetrically into the bcc single phase region.

We propose a new explanation of this state of two-phase equilibrium by extending the polymorphic transformation concept to include defects in the alloy. It is not surprising that a higher average defect concentration can explain the loss of stability of the bcc phase with milling intensity. To explain two-phase coexistence, however, we consider heterogeneities in the defect concentration in the alloy. We propose that near the polymorphic transformation composition, the most defective bcc crystallites will transform into fcc crystallites, even if the chemical contribution to the free energy for the bcc phase is favorable. Owing to heterogeneities in the defect density in ball-milled material, the free energy versus composition curves for both the fcc and bcc phases are no longer sharp lines, but are effectively smeared upwards in free energy. Using model free-energy calculations, 
we show that the region of two-phase coexistence can be predicted if ball milling creates heterogeneities of $2-3 \mathrm{~kJ} /$ mole in the free energy of the bcc phase. We find that this defect enthalpy is consistent with the average heat release measured by differential scanning calorimetry. Our interpretation of the region of two-phase coexistence in ball-milled materials is essentially the same as that used for understanding Monte Carlo simulations of bcc alloys with thermal and ballistic atom movements. ${ }^{58,59}$

\section{EXPERIMENT}

\section{A. Milling conditions}

Appropriate masses of elemental $\mathrm{Fe}$ and $\mathrm{Ni}$ powders with $99.9 \%$ purity were mixed and sealed in a steel vial under an argon atmosphere. In what follows, all compositions are given in atomic percent, and we use " $x$ " to designate the atomic percentage of $\mathrm{Ni}$. Ball milling was performed with a Spex 8000 mixer/mill using hardened steel vials and balls, with a ball-to-powder weight ratio of 5:1. The temperature of the vial was kept below $60{ }^{\circ} \mathrm{C}$ during milling by forced air cooling. After the milling was stopped, the vial was cooled for a few hours before opening. Using four steel balls (two $10 \mathrm{~mm}$, two $3 \mathrm{~mm}$ ), the standard Spex 8000 mill provides a milling intensity that we denote as $I_{1}$. With $I_{1}$ the ball velocity has been reported to be about $3 \mathrm{~m} / \mathrm{s}^{60}$ In a series of preliminary tests, we found that milling for $24 \mathrm{~h}$ at the $I_{1}$ intensity was more than sufficient time to reach a steady-state microstructure; no differences in grain size or phase fraction were detected when the powders were milled for more than about $12 \mathrm{~h}$. To test the effect of milling intensity on the steady-state microstructures of the powders, we modified the Spex mill to operate at a second milling intensity, lower than the standard one. We replaced the belt drive pulley on the shaker with a new one having an effective diameter $\sqrt{3}$ as large as the commercial pulley. This modification should reduce the kinetic energy of the balls to approximately one-third of the kinetic energy for intensity $I_{1}$. We denote this lower ball milling intensity as $I_{0.3}$. We found that $48 \mathrm{~h}$ of continuous milling was sufficient for the powders to reach steady states with this lower intensity $I_{0.3}$. Our interest in the present experiments was to study the steadystate microstructures produced by ball milling, not the kinetics of achieving them. All milling with intensity $I_{1}$ was performed for $24 \mathrm{~h}$, and all milling with intensity $I_{0.3}$ was performed for $48 \mathrm{~h}$.

During milling there was some tendency for the powders to become enriched with Fe from the vials and balls. Chemical analyses of several of the as-milled powders were performed by atomic emission spectrometry, where the powder was dissolved in acid solution, introduced as an aerosol in an inductively coupled plasma discharge, and the light emission analyzed by an optical spectrometer. We found that the powders milled at the higher intensity $I_{1}$ increased their Fe concentration more than did the samples milled at the $I_{0.3}$ intensity; typical changes in concentration were 3 at. $\%$ for $I_{1}$ and 0.2 at. $\%$ for $I_{0.3}$. The chemical concentrations given below were corrected for this $\mathrm{Fe}$ contamination.

\section{B. X-ray diffractometry}

X-ray diffractometry was performed on the as-milled powders with an Inel CPS-120 diffractometer system (Debye-Scherrer optics) having a curved position sensitive detector (PSD) spanning $127^{\circ}$ in $2 \theta$ angle. The Co $K \alpha$ incident radiation was monochromated with a flat graphite monochromater. We determined the fractions of bcc and fcc phases in the alloys by comparing the intensities of the bcc(200) and fcc(220) peaks, which were reasonably immune to problems of overlap with broadened neighboring peaks. Detection limits for the bcc and fcc phases were set primarily by the statistical quality of our data, and are estimated to be about $0.5 \%$.

We corrected the measured peak intensities, $I(\theta)$, for Lorentz polarization, multiplicity, and structure factors in a standard way. ${ }^{61}$ Absorption corrections for our flat samples with Debye-Scherrer optics differ from those for a BraggBrentano diffractometer, however, ${ }^{62}$ and require knowledge of the angle of incidence, $\phi$, for the x-ray beam on the flat sample, which was $15^{\circ}$. The corrected peak intensity, $I_{\text {cor }}$, was

$$
\begin{aligned}
I_{\text {cor }}(\theta)= & \frac{\sin \theta \sin 2 \theta}{1+\cos ^{2} \theta} \frac{V_{c}^{2} \mu \rho}{m F^{*}(k) F(k)} \\
& \times \frac{\sin \phi+\sin (2 \theta-\phi)}{\sin (\theta-\phi)} I(\theta),
\end{aligned}
$$

where $V_{c}$ is the volume of the unit cell, $\mu$ is the mass absorption coefficient, $\rho$ is the density of the material, $m$ is the multiplicity of the diffracting planes, and $F(k)$ is the structure factor for the unit cell. As usual, $k=4 \pi \sin \theta / \lambda$. Our samples showed little if any crystallographic texture, so the fractions of constituent phases, $f_{\text {bcc }}$ and $f_{\text {fcc }}$, were determined by taking ratios of the corrected intensities of the bcc(200) and fcc(220) diffractions

$$
\frac{f_{\text {bcc }}}{f_{\text {fcc }}}=\frac{I_{\text {cor } 200}}{I_{\text {cor } 220}} .
$$

For nanocrystalline alloys, the absorption factors, $\mu \rho$, are the same for both bcc and fcc phases, and therefore cancel in Eq. (2). We also assume the same atomic form factors for the atoms in the fcc and bcc phases. Equation (2) becomes

$$
\begin{aligned}
& \frac{f_{\mathrm{bcc}}}{f_{\mathrm{fcc}}}=1.657 \frac{I_{200}}{I_{220}}, \\
& f_{\mathrm{bcc}}=\frac{1.657 I_{200}}{1.657 I_{200}+I_{220}}, \\
& f_{\mathrm{fcc}}=\frac{I_{220}}{1.657 I_{200}+I_{220}} .
\end{aligned}
$$

Two types of x-ray peak broadening analysis were used to obtain the average grain size and root-mean-squared strain. In the method of Williamson and Hall, ${ }^{63,64}$ we measured and graphed the diffraction linewidth in $k$-space versus the $k$-vector of four diffraction peaks. We obtained the grain size from the extrapolated $y$-intercept, and the root-mean- 
squared strain from the slope of the plot. In a second method for obtaining grain size data, we analyzed the widths of the bcc(110) and fcc(111) diffractions with the Scherrer equation ${ }^{65,66}$

$$
L=\frac{0.9 \lambda}{B(2 \theta) \cos \theta},
$$

where $B(2 \theta)$ is the full width at half maximum (in radians) of a Lorentzian function fit to the diffraction peak. Our previous work showed reasonable agreement between the grain size obtained from Eq. (5) and the grain size obtained from dark field imaging with a transmission electron microscope. ${ }^{67,68}$ Lattice parameters for the fcc and bcc phases were determined by the Nelson-Riley extrapolation method, ${ }^{69,70}$ with calibration provided by a powdered Si standard.

\section{Mössbauer spectrometry}

Mössbauer spectra were measured at room temperature in transmission geometry with a conventional constant acceleration spectrometer using a $40 \mathrm{mCi}{ }^{57} \mathrm{Co}$ in $\mathrm{Rh}$ radiation source. The ${ }^{57} \mathrm{Fe}$ hyperfine magnetic field distribution is sensitive to the local chemical environment of the ${ }^{57} \mathrm{Fe}$ atom. ${ }^{71,72}$ and was useful for chemical analysis of the bcc phase in our $\mathrm{Fe}-\mathrm{Ni}$ alloys. The average hyperfine magnetic field was obtained from the spectrum by fitting the first and sixth peaks of the magnetic sextet to Lorentzian functions, and comparing the splitting of these two peaks to the splitting of the first and sixth peaks measured from bcc Fe, which is known to be $330 \mathrm{kG}$ at room temperature. Chemical analysis of the bcc phase was based on comparing the measured hyperfine magnetic fields to those of standard samples of known composition. ${ }^{72}$ To obtain additional calibration points, we also made three $\mathrm{Fe}-\mathrm{Ni}$ ingots of $\mathrm{Ni}$ concentrations, $x=15,20,25$, by arc melting under an Ar atmosphere. These ingots were inverted and remelted four times, and filings taken from them were found to be bcc by x-ray diffractometry. Mössbauer spectra were measured from these filings.

\section{Differential scanning calorimetry}

Differential scanning calorimetry (DSC) was performed in a flowing helium atmosphere with a Perkin-Elmer DSC-7 instrument over the temperature range of $50-550{ }^{\circ} \mathrm{C}$. Powdered samples were sealed in vented pans made of annealed aluminum, and run against a matched empty pan for reference. For each sample, two identical heating runs at 20 $\mathrm{K} /$ min were performed in sequence, so the recovery of defect structures and grain growth that occurred in the first run could be identified from the difference of these two runs. The DSC-7 was calibrated with a NIST sapphire standard.

\section{RESULTS}

\section{A. Phase fractions}

Figure 1 presents $\mathrm{x}$-ray diffraction patterns from powders of $\mathrm{Fe}_{91} \mathrm{Ni}_{9}, \mathrm{Fe}_{77} \mathrm{Ni}_{23}$, and $\mathrm{Fe}_{63} \mathrm{Ni}_{37}$ ball milled at $I_{1}$ for $24 \mathrm{~h}$. While the diffraction pattern from $\mathrm{Fe}_{77} \mathrm{Ni}_{23}$ has both bcc and fcc peaks, the $\mathrm{Fe}_{91} \mathrm{Ni}_{9}$ pattern has only bcc peaks and the $\mathrm{Fe}_{63} \mathrm{Ni}_{37}$ pattern has only fcc peaks. The bcc and fcc

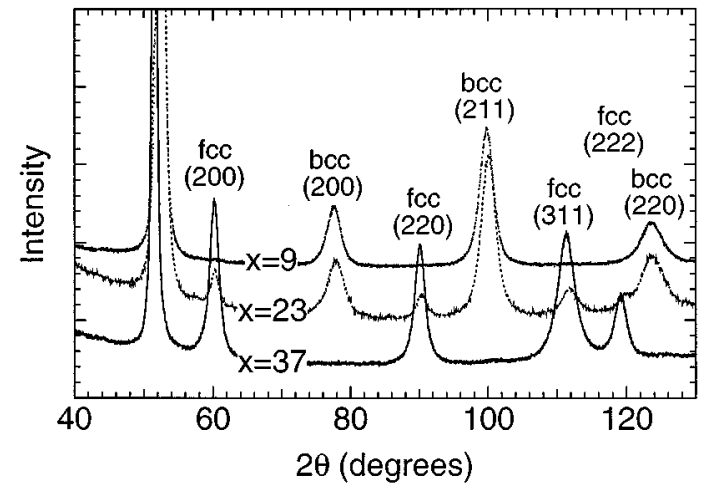

FIG. 1. X-ray diffraction patterns from as-milled $\mathrm{Fe}_{100-x} \mathrm{Ni}_{x}$ alloys with $x=9,23,37$. Materials were milled for $24 \mathrm{~h}$ with intensity $I_{1}$.

phase fractions were calculated according to Eqs. (4), and are plotted against the alloy Ni concentration $x$ in Fig. 2. Compared to the bounderies from the equilibrium phase diagram at low temperatures, the bcc and fcc single phase regions are extended considerably. For example, at $60{ }^{\circ} \mathrm{C}$ the equilibrium two-phase region extends approximately from $2 \% \mathrm{Ni}$ to $71 \% .^{73}$ We determined the compositional width of the twophase region in the as-milled materials by extrapolation of the data in Fig. 2 to zero concentration of the phases, and found the boundaries to be at $x \approx 12$ and 30 for $I_{1}$ and $x \approx 18$ and 34 for $I_{0.3}$, respectively. Higher milling intensity shifts the phase boundaries asymmetrically toward the bcc region, with the bcc phase boundary being shifted most strongly. Furthermore, for any Ni concentration, the higher ball milling intensity, $I_{1}$, always results in less bcc phase than the lower milling intensity, $I_{0.3}$.

\section{B. Chemical analysis}

For $\mathrm{Fe}_{100-x} \mathrm{Ni}_{x}$ alloys ball milled at $I_{1}$ for $24 \mathrm{~h}$, we observed essentially pure bcc phase for $x \leqslant 12$ and pure fcc phase for $x \geqslant 30$. We therefore expect that ball milling extends the solubility of $\mathrm{Ni}$ in the bcc phase to at least $12 \%$, and the Fe concentration in the fcc phase is extended to at least $70 \%$. We have evidence, however, that in the two-phase

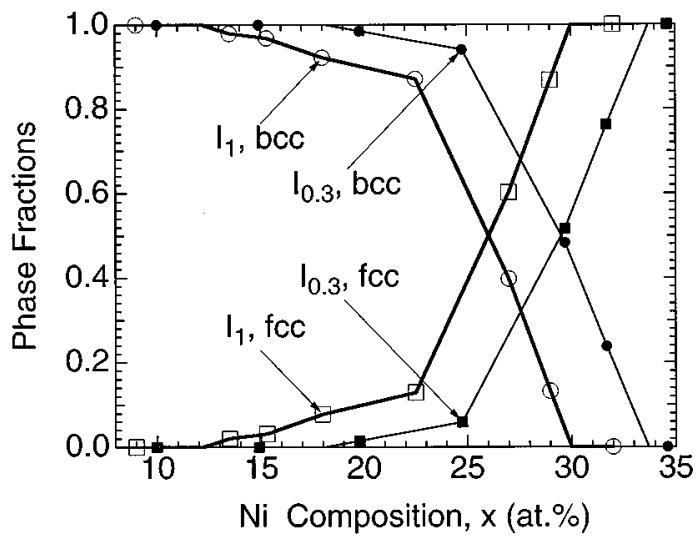

FIG. 2. Fractions of bcc and fcc phases in as-milled $\mathrm{Fe}_{100-x} \mathrm{Ni}_{x}$ alloys, determined by $\mathrm{x}$-ray diffractometry. Alloys were milled for $24 \mathrm{~h}$ with intensity $I_{1}$, and for $48 \mathrm{~h}$ with intensity $I_{0.3}$. 


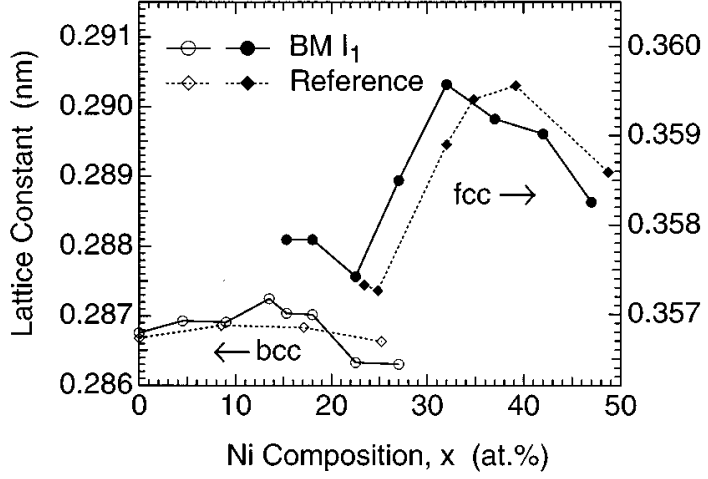

FIG. 3. Lattice parameters of bcc and fcc phases in as-milled $\mathrm{Fe}_{100-} \mathrm{Ni}_{x}$ alloys. Data from Refs. 74 (fcc), and 75 (bcc), are also shown.

region the chemical compositions of the individual bcc and fcc crystallites are extended even further. X-ray lattice parameter data are presented in Fig. 3. Figure 3 also includes results from the JCPDS index of bcc and fcc alloys, and other previously reported data. ${ }^{74,75}$ There is excellent agreement between the lattice parameter data from our bcc powders and from those reported previously, suggesting that the single-phase as-milled bcc powders do not have a significant mean strain. The data of Fig. 3 suggest a mean strain of about $0.1 \%$ for the Ni-rich single-phase fcc powders, however. In the two-phase region for $\mathrm{Ni}$ concentrations below $30 \% \mathrm{Ni}$, the lattice parameters of the fcc phase decrease significantly below their values in single-phase $\mathrm{Fe}-\mathrm{Ni}$. In the composition range of two-phase coexistence, the lattice parameters of as-milled $\mathrm{Fe}-\mathrm{Ni}$ follow closely the lattice parameters of bulk $\mathrm{Fe}-\mathrm{Ni}$ alloys of nearly the same chemical composition. The lattice parameters of the fcc phase in the twophase region are not characteristic of an alloy having a chemical composition of $30 \% \mathrm{Ni}$, which marks the boundary of the fcc single-phase region. Unfortunately, the lattice parameter of the bcc phase is rather insensitive to the chemical composition of the alloy, so the bcc lattice parameter data were not useful for checking the chemical composition of the bcc phase.

Average hyperfine magnetic fields obtained from Mössbauer spectrometry are presented in Fig. 4, together with some hyperfine magnetic fields reported previously for bcc $\mathrm{Fe}-\mathrm{Ni}$ alloys, ${ }^{72}$ and data from our own bcc calibration samples. At least to compositions of $12 \%$ or so, these data show that the Ni concentration in the bcc phase of the asmilled powder is the same as the overall alloy composition. Unfortunately, for higher $\mathrm{Ni}$ concentrations the hyperfine magnetic field becomes insensitive to the Ni concentration in the bcc phase, and is not a useful measure of chemical composition. The measured hmf's are nevertheless consistent with the x-ray result that the chemical composition the bcc phase is nearly the same as the overall composition of the alloy.

\section{Defect enthalpy}

The shapes of x-ray diffraction peaks are shown in Fig. 5 for the $\mathrm{Fe}_{100-x} \mathrm{Ni}_{x}$ powders ball milled with intensities $I_{1}$ and

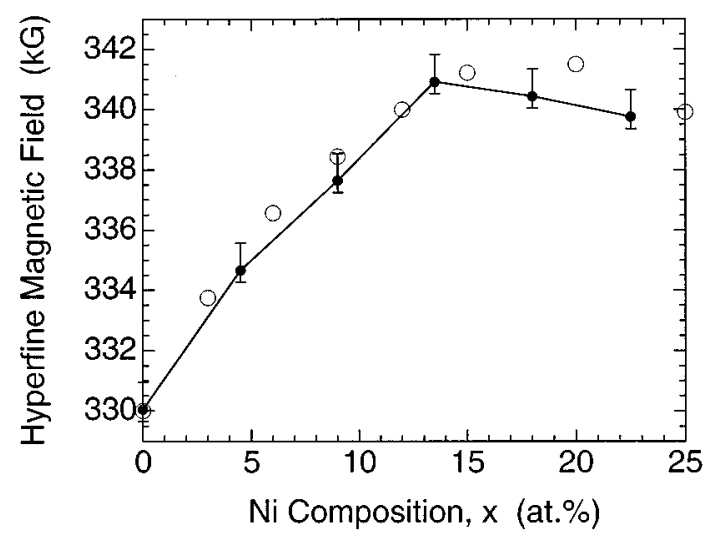

FIG. 4. ${ }^{57} \mathrm{Fe}$ hyperfine magnetic fields for the bcc phase of as-milled $\mathrm{Fe}_{100-x} \mathrm{Ni}_{x}$ alloys. Also shown are hyperfine magnetic fields from previous work (Ref. 72), and data points from bulk bcc alloys obtained in the present study.

$I_{0.3}$. These diffraction peaks were normalized to give the same backgrounds and the same bcc (200) peak heights [Fig. $5(\mathrm{a})]$ or the same fcc(220) peak heights [Fig. 5(b)]. Figures 5(a) and 5(b) show that the diffraction peak shapes for the bcc and fcc phases were little changed for the different compositions and milling conditions. (Artifacts of the positionsensitive detector have distorted the shapes of the weakest diffraction peaks.) These diffraction peaks have significant intensities in their tails, typical of a particle size distribution that includes some small crystallites. The average grain size and root-mean-squared strain are presented as functions of nickel composition in Figs. 6(a) and 6(b), respectively. Within the capabilities of our peak analysis methods we do not find any differences in the grain size or internal strain distribution caused by milling intensity or by chemical composition differences. We do find, however, that the bcc phase has both smaller grains and a larger root-mean-squared strain than does the fcc phase. This is not surprising and is consistent with previous studies. ${ }^{38,39}$

The DSC traces presented in Fig. 7 are differences between the first and second runs of the sample to $550{ }^{\circ} \mathrm{C}$. For all specimens we observed a broad exothermic peak that began at $130{ }^{\circ} \mathrm{C}$. We also observed a second peak at about $400{ }^{\circ} \mathrm{C}$ for the bcc alloys and $520^{\circ} \mathrm{C}$ for the fcc alloys. We performed $\mathrm{x}$-ray diffraction measurements on the powders heated in the DSC to $550^{\circ} \mathrm{C}$. In none of the samples of pure bcc or fcc phase did we observe any transformation to the other phase after the DSC scans. We therefore interpret the measured heats as enthalpies of defect recovery. We also performed partial DSC scans to $300{ }^{\circ} \mathrm{C}$, which is a temperature between the first and second broad peaks in Fig. 7. From an $\mathrm{x}$-ray lineshape analysis with the Williamson-Hall method, we found that the first peak corresponded primarily to a relaxation of the root-mean-squared strain, whereas the second peak corresponded to grain growth. Integrating the DSC traces such as those in Fig. 7 gave about $1.5 \mathrm{~kJ} / \mathrm{mol}$ for the bcc alloys, and $0.5 \mathrm{~kJ} / \mathrm{mol}$ for the fcc alloys. In both cases the integrated heat in the first peak was approximately 

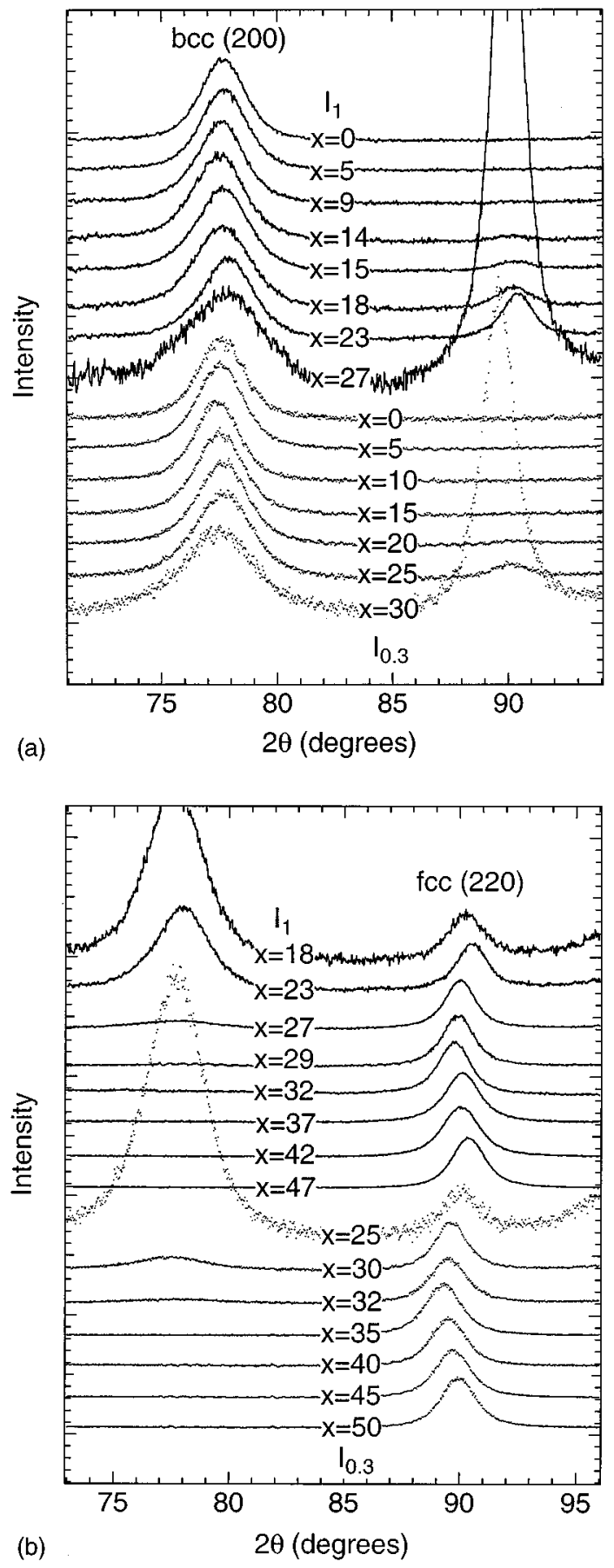

FIG. 5. X-ray diffraction peak shapes: (a) (200) bcc and (b) (220) fcc. Peaks are presented with background corrections and normalized intensities.

the same as the integrated heat in the second peak. These numbers are only approximate, however. We hesitate to claim, for example, that the materials milled at the lower intensity showed a smaller heat evolution than those milled at the higher intensity, although this seems to be true. Unfortunately, in the composition range from $25<x<35$, the fcc phase undergoes a ferromagnetic Curie transition in the temperature range of our DSC measurements. We were therefore unable to extract reliable enthalpies of defect recovery for the two-phase alloys.

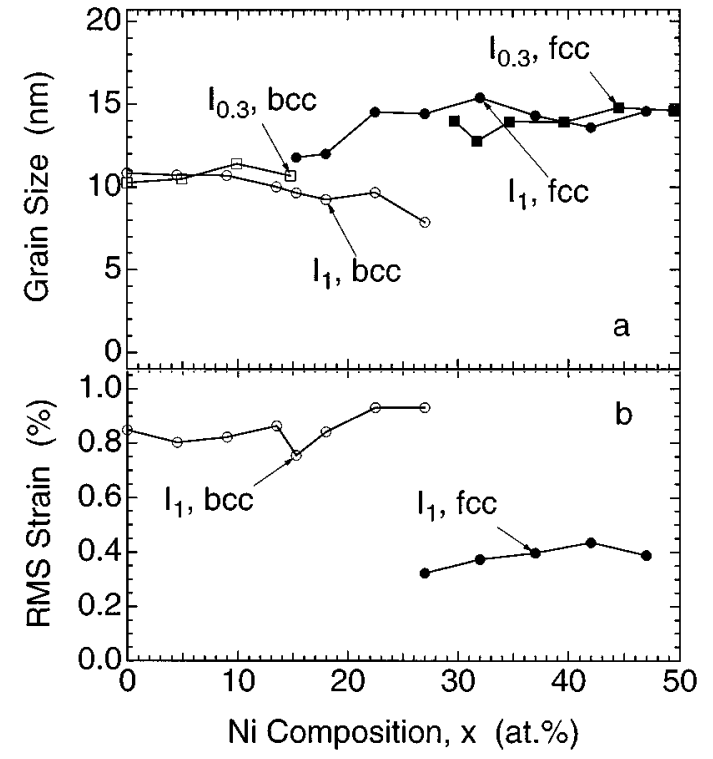

FIG. 6. X-ray diffractometry determinations of grain size and root-meansquared strain of the bcc and fcc phases of as-milled $\mathrm{Fe}_{100-x} \mathrm{Ni}_{x}$ alloys. (a) Grain size determined by Scherrerr broadening of (110) bcc and (111) fcc diffractions, and (b) root-mean-squared strain determined by the method of Williamson and Hall (Ref. 63).

\section{DISCUSSION}

\section{A. Features of two-phase coexistence}

For Fe-rich alloys, the as-milled alloy is fully bcc. For $\mathrm{Ni}$-rich alloys, the as-milled alloy is fully fcc. At intermediate compositions, however, the as-milled alloy is a two-phase mixture of bcc and fcc phases. This two-phase region spans the range in $\mathrm{Ni}$ concentration, $18<x<34$, for the milling intensity $I_{0.3}$, and the broader composition range, $12<x<30$, for the intensity $I_{1.0}$. Recall that conventional thermodynamic explanations of two-phase coexistence rely on chemical segregation between the two phases. The minimum in total free energy of a two-phase alloy is found by constructing a common tangent between the free energy curves of the two phases. The points of tangency mark the equilibrium compositions of the two phases for any alloy having a composition between these two compositions.

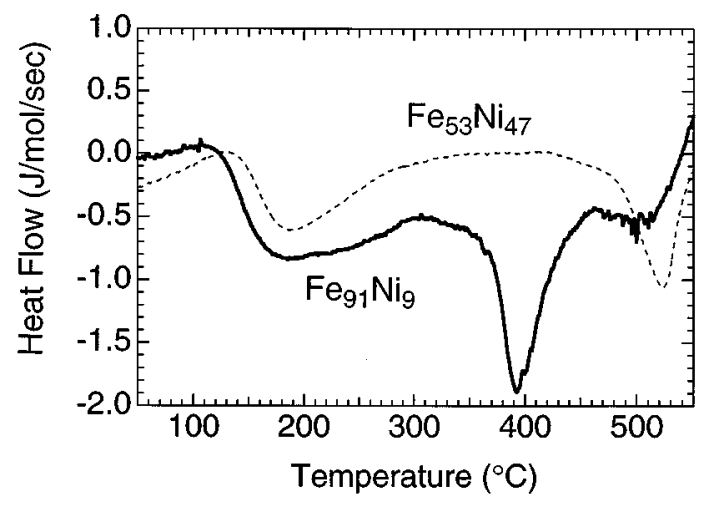

FIG. 7. DSC scans of $\mathrm{Fe}_{89} \mathrm{Ni}_{9}$ and $\mathrm{Fe}_{63} \mathrm{Ni}_{37}$ milled at intensity $I_{1}$ for $24 \mathrm{~h}$, after subtracting background obtained from a subsequent scan. 
For several reasons we doubt that the two-phase coexistence found in our as-milled alloys can be understood with the conventional thermodynamic explanation. There is poor agreement between the phase fractions shown in Fig. 2 and the predictions with the lever rule and the $\mathrm{Fe}-\mathrm{Ni}$ phase diagram for any assumed temperature. Even allowing for enhanced solid solubility in the bcc and fcc phases, the conventional argument is unsuccessful in predicting the trends of Fig. 2-the lever rule predicts a linear change in phase fraction across the two-phase region, which is inconsistent with the strongly skewed curves of Fig. 2.

Another reason we doubt the conventional thermodynamic explanation of two-phase coexistence is our evidence that through the two-phase region the compositions of the bcc and fcc phases are approximately the same. In particular, our data on lattice parameters show that in the two-phase region, the fcc phase has a chemical composition close to that of the alloy itself. It is difficult to determine a precise chemical composition of a phase from its lattice parameter, but the data of Fig. 3 suggest that the fcc phase is enriched in Ni by perhaps only $3 \%$ beyond the overall composition of the alloy. By conservation of solute, the bcc phase must also have nearly the composition of the alloy. Although not sufficient in themselves, our data on hyperfine magnetic fields are consistent with the bcc phase having the composition of the bulk alloy. Our data cannot show an absence of chemical segregation to much better than $3 \%-4 \%$, but this accuracy is sufficient for what follows. A previous study of as-milled alloys of $\mathrm{Fe}-\mathrm{Cu}$ suggested that both the bcc and fcc phases had nearly the same compositions, ${ }^{38}$ and we note that the elements $\mathrm{Fe}$ and $\mathrm{Cu}$ are less miscible than $\mathrm{Fe}$ and $\mathrm{Ni}$.

Another peculiarity of the two-phase region is that it becomes broader as the milling intensity is increased. This is inconsistent with the general expectation that a higher ballmilling intensity should promote interatomic mixing and enhance solid solubility. Notice that the broadening of the twophase region is asymmetrical; with higher milling intensity the fcc single-phase region grew, but the bcc single-phase region shrank more.

\section{B. Interpretations of two-phase coexistence}

Using results from previous assessments of the $\mathrm{Fe}-\mathrm{Ni}$ phase diagram implemented by the commercial software package Thermo-Calc, ${ }^{76,77}$ the Helmholtz free energy versus composition was calculated for bcc and fcc $\mathrm{Fe}-\mathrm{Ni}$ alloys at various temperatures, $F_{\mathrm{bcc}}(x, T)$ and $F_{\mathrm{fcc}}(x, T)$. The dark curves in Fig. 8 are the calculations for $F_{\mathrm{bcc}}$ and $F_{\mathrm{fcc}}$ at 300 $\mathrm{K}$. These results were obtained by extrapolation of higher temperature data, so their precise reliability is unknown. Nevertheless, it is interesting that the two curves cross at $x=28$, which is quite close to the compositions where the as-milled alloys have equal fractions of the bcc and fcc phases ( $x=26$ and 30 in Fig. 2). This crossing of free energy curves defines the polymorphic transformation composition at the temperature of interest. (We assume this temperature is $300 \mathrm{~K}$, but Thermo-Calc results show that the polymorphic transformation composition does not vary strongly with temperature; it decreases by only $4 \% \mathrm{Ni}$ when the temperature is raised from $300 \mathrm{~K}$ to $500 \mathrm{~K}$.) Polymorphic transformations

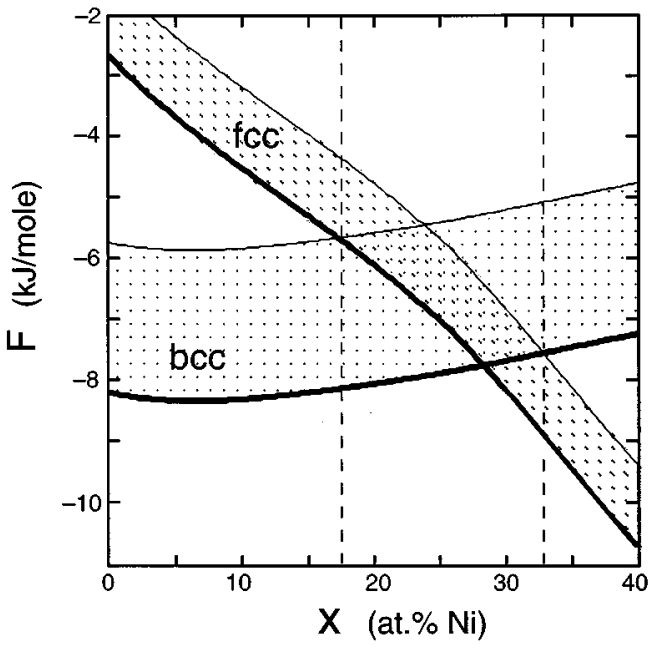

FIG. 8. Dark curves: Thermo-Calc calculations of the free energies of bcc and fcc $\mathrm{Fe}-\mathrm{Ni}$ alloys at $300 \mathrm{~K}$ (reference states for $\mathrm{Fe}$ and $\mathrm{Ni}$ were their stable element reference states at $298 \mathrm{~K}$ and $1 \mathrm{~atm}$ ). For description of bands above the dark curves, see Sec. IV B.

involve a change in structure with no change in composition, so it is not surprising that the polymorphic transformation line is relevant for as-milled alloys. A similar argument was used previously for understanding extended solid solubility in $\mathrm{Fe}-\mathrm{Cu}^{38-44,47}$

A simple crossing of free-energy curves cannot explain two-phase coexistence, however. For compositions on either side of the crossing point of the two curves, the free energy is minimized by the existence of only one phase. We have considered modifications of the polymorphic transformation concept to explain two-phase coexistence in ball-milled $\mathrm{Fe}-$ $\mathrm{Ni}$. These modifications presume that the alloy has spatial heterogeneities involving (i) composition, (ii) temperature, (iii) pressure, and (iv) defect structures. In the present section we discuss in sequence the success of these ideas.

Compositional heterogeneities would allow the Ni-rich regions to be fcc, and $\mathrm{Ni}$-poor regions to be bcc, based on the free-energy curves $F_{\text {fcc }}\left(x, T^{\prime}\right)$ and $F_{\mathrm{bcc}}\left(x, T^{\prime}\right)$. The problem with this argument is that rather large compositional heterogeneities are required to explain the data of Fig. 2. For example, in alloys with low $\mathrm{Ni}$ concentration this argument predicts that the fcc regions would have $\mathrm{Ni}$ enrichments of more than $15 \%$, and our lattice parameter trend is inconsistent with such large chemical heterogeneities. A second problem with the concept of compositional heterogeneities is that we would expect the heterogeneities to be suppressed with higher milling intensity. With higher milling intensity, however, the composition range for two-phase coexistence grows larger, in contrast to this expectation.

The existence of thermal heterogeneities can cause twophase coexistence if the polymorphic transformation composition has a strong temperature dependence. For the bcc and fcc phases of $\mathrm{Fe}-\mathrm{Ni}$, however, Thermo-Calc calculations show that the polymorphic transformation line is nearly vertical (see, also, Fig. 7 in Ref. 78). Since the polymorphic transformation composition changes little with temperature, temperature elevations of $500 \mathrm{~K}$ would be needed to obtain 
fcc phase in the Ni-poor alloys. Although it could be argued that such local temperature variations are induced by ball milling, the bcc phase could not be obtained in the Ni-rich alloys for any elevation of temperature.

Owing to the volume difference of $3 \%$ between the bcc and fcc phases, pressure heterogeneities will affect strongly the thermodynamic stabilities of the bcc and fcc phases. Since the bulk moduli of both phases are high, high elastic energies can be achieved with modest internal strains. An elastic strain of $1 \%$ can increase the free energy of the bcc phase by several $\mathrm{kJ} / \mathrm{mole}$, so reasonable strains can cause large shifts of free energy curves. There is certainly a distribution of internal strains in the as-milled alloys, as shown by the data of Fig. 6(b), and these internal strains are larger for the bcc phase than for the fcc phase. Since the bcc phase is also expected to have the larger elastic moduli, it has a wider distribution of elastic energy density. We believe that these localized strain distributions, which originate with dislocations and other defect structures, have major effects on the stability of individual bec and fcc crystallites.

We also expect the stability of bcc or fcc crystallites to be affected by the high density of grain boundaries, which are capable of elevating bulk enthalpies by several $\mathrm{kJ} / \mathrm{mole}^{13}$ The grain boundary energy cannot be understood easily as a property of one crystallite or another, but this is not necessary. Regions rich in small bcc crystallites, for example, will be expected to have a higher internal energy than will regions rich in larger fcc crystallites.

We do not know accurately the relative importances of grain boundaries and other defects in affecting the free energy, although the two peaks in the DSC traces, the strain recovery peak and the grain growth peak, have about equal areas. Similarly, our x-ray lineshape analysis does not provide precise numbers for the grain sizes and the root-meansquared strains. Nevertheless, we can state with confidence that the enthalpy measured by DSC scans of the bcc material (about $1.5 \mathrm{~kJ} / \mathrm{mol}$ ) is greater than that of the fcc material (about $0.5 \mathrm{~kJ} / \mathrm{mol}$ ), and the $\mathrm{x}$-ray peaks from the bcc phase are broadened more strongly than the fcc peaks. Besides the larger defect enthalpy in the bcc phase, we have the following evidence that there is a larger distribution in defect enthalpy in the bcc phase. Dark field transmission electron microscopy studies of grain size distributions in ball-milled alloys have shown a broad distribution of crystallite sizes, ${ }^{67,68}$ and this is consistent with the tendency of diffraction peaks from ball milled materials to have Lorentzian shapes. ${ }^{79}$ Our x-ray diffraction peaks were not accurately Lorentzian functions, but they did show strong intensities in their tails, and more so for the bcc alloys. The root-meansquared strain measured for the bcc alloys, which was about twice as large as for the fcc alloys, originates from a large distribution of local interplanary spacings in the alloy.

With a distribution of grain sizes and a distribution of internal strains, we expect that different crystallites will be destabilized by different amounts. In what follows, we consider in a general sense the heterogeneities in free energies of the bcc and fcc phases in $\mathrm{Fe}-\mathrm{Ni}$ alloys prepared by ball milling. The free energy is considered in the sense of a "coarse-grained" free energy, where the coarse graining en- compasses several crystallites. The meaning of the internal energy is not a serious problem in principle, since we can add to the internal energy new contributions from defect density and grain size. The magnitude of the excess entropy induced by ball milling is, however, less clear. The simple approach is to ignore it, and we justify this as follows. Microstructural features such as dislocations and grain boundaries are often termed "nonthermodynamic," because their abundance in thermodynamic equilibrium is essentially negligible. This can be true only if they are responsible for a relatively minor increase in the entropy of the alloy, but a relatively large increase in enthalpy. To a first approximation, our picture of an increased free energy in ball-milled materials reflects primarily an increase in the internal energy due to the microstructural defects created by milling.

To accommodate the spatial heterogeneities in coarsegrained free energy, we modify Fig. 8 by drawing a band above the calculated dark curves for $F_{\mathrm{bcc}}$ and $F_{\mathrm{fcc}}$. The bands serve to indicate approximately the distribution of free energy that exists for fcc and bcc regions of differing defect densities. Since the x-ray diffraction peak shapes do not depend on composition for either the bcc or fcc phases (Figs. 5 and 6), we have represented the elevation of the free-energy curves as equal vertical shifts at all compositions. The centers of the bands match the average enthalpy measured from DSC scans of bcc and fcc material. In milled materials we might expect upwards translations of the free-energy bands that will contribute to the heat evolution measured in a DSC experiment. However, the lattice parameters of Fig. 3 agree reasonably well with those of bulk $\mathrm{Fe}-\mathrm{Ni}$ alloys, ${ }^{74,75}$ indicating that there is no large average strain in the material. It seems plausible that the bottoms of the free-energy bands, associated with the least defective regions, are not altered significantly by ball milling.

During milling, we assume it is possible for a crystallite of one phase to transform into a crystallite of the other phase when the local coarse-grained free energy favors the transformation. (There may be a nucleation impediment to this transformation, but nucleation should be less of a problem when both phases are already present in the alloy, or when martensitic transformations can occur.) Such a transformation could occur if the chemical contribution to the free energy is not favorable, provided that the defect enthalpy is reduced significantly by the transformation. Such transformations of the more defective crystallites would be expected to occur in the composition range from $15<x<33$, where the free energy bands for the fcc and bcc phases overlap in Fig. 8. We have drawn vertical dashed lines in Fig. 8 to indicate the expected two-phase region. We expect that for compositions $x<15$, the chemical preference for the bcc phase is too large to be overcome by milling-induced defects. Similarly for $x>33$, the defect density in the fcc phase is insufficient for fcc crystallites to be destabilized with respect to perfect bcc crystallites.

The overlap of the free-energy bands in Fig. 8 explains fairly well the two-phase coexistence shown in Fig. 2. A little more detail is required to explain the asymmetrical phase fraction curves in Fig. 2 (i.e., the negative skewness for the fraction of fcc phase). We propose two reasons for this asym- 


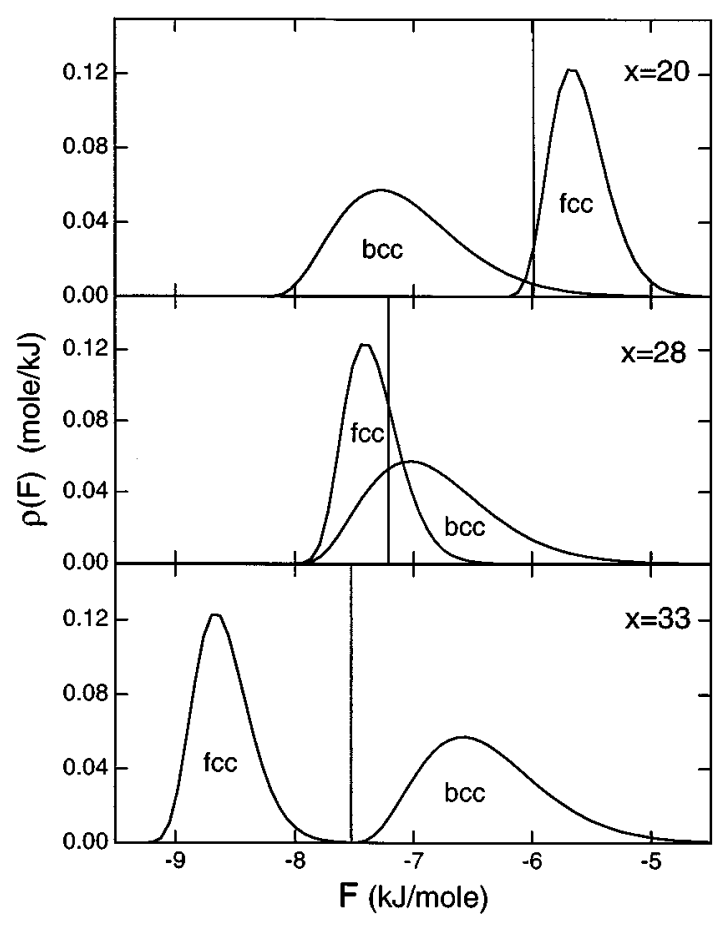

FIG. 9. Semiquantitative distributions of free-energy functions for bcc and fcc $\mathrm{Fe}-\mathrm{Ni}$ alloys of three compositions at $300 \mathrm{~K}$ after ball milling. The lowest energies of each curve were obtained from the dark curves of Fig. 8 for compositions $x=20,28$, and 33 .

metry. Differences in curvature of the Thermo-Calc free energy curves of the bcc and fcc phase (see Fig. 8) cause the bcc and fcc free-energy bands to approach each other on the Fe-rich side of the polymorphic transformation composition. The increased overlap of these bands will extend the range of fcc phase to lower Ni concentrations. Our second proposed reason for the asymmetrical phase fraction curves in Fig. 2 involves asymmetrical distribution functions for the coarsegrained free energies of the fcc- and bcc-rich regions. Figure 9 shows the type of free-energy distribution functions that are needed to predict the skewness of the phase fraction data of Fig. 2. (For conventional thermodynamics as in ThermoCalc calculations, the distribution functions in Fig. 9 would be Dirac delta functions whose positions change with composition.) We have drawn a positive skewness of the distribution functions in Fig. 9, and a wider spread of the bcc free-energy distribution function, $\rho_{\mathrm{bcc}}(F, x, T=300 \mathrm{~K})$, compared to the fcc free-energy distribution function, $\rho_{\mathrm{fcc}}(F, x, T=300 \mathrm{~K})$. Each of the distribution functions has been normalized to unity

$$
\begin{aligned}
& 1=\int_{-\infty}^{\infty} \rho_{\mathrm{bcc}}\left(F, x^{\prime}, T^{\prime}\right) d F, \\
& 1=\int_{-\infty}^{\infty} \rho_{\mathrm{fcc}}\left(F, x^{\prime}, T^{\prime}\right) d F .
\end{aligned}
$$

A two-phase alloy need not have regions that fill all states in $\rho_{b c c}$ and $\rho_{\mathrm{fcc}}$, however. Instead, we expect that the states to be filled approximately to a maximum, $F_{F}$, defined [this argument runs parallel to the using a density of states function for electrons, $\rho(E)$, that is filled to a Fermi level, $E_{F}$, defined at low temperature as the energy that accounts for all electrons] so that

$$
1=\int_{-\infty}^{F_{F}} \rho_{\mathrm{fcc}}\left(F, x^{\prime}, T^{\prime}\right)+\rho_{\mathrm{bcc}}\left(F, x^{\prime}, T^{\prime}\right) d F .
$$

Vertical lines mark the values of $F_{F}$ for the three cases shown in Fig. 9. Note in particular that at the composition corresponding to the crossover of the free-energy curves $(x$ $=28$ ), a greater fraction of fcc phase is expected than bcc phase. By shifting the bottoms of the distribution functions in Fig. 9 to coincide with the compositions provided by the Thermo-Calc free-energy curves of Fig. 8, the asymmetry of the phase fraction data of Fig. 2 is predicted qualitatively.

The prediction of the asymmetrical phase fraction curves in Fig. 2 requires that $\rho_{\mathrm{bcc}}\left(F, x^{\prime}, T^{\prime}\right)$ has a tail that extends to higher $F$ than the tail of $\rho_{\text {fcc }}\left(F, x^{\prime}, T^{\prime}\right)$. With increased milling intensity, we expect this tail of $\rho_{\mathrm{bcc}}\left(F, c^{\prime}, T^{\prime}\right)$ to extend to yet higher $F$. We can then understand how with increased milling intensity, the two-phase region should extend further into the region of low Ni concentration because $F_{F}$ moves upwards to include relatively more of the fcc phase. The effect of milling intensity on the free-energy distribution of the fcc phase, $\rho_{\mathrm{fcc}}\left(F, c^{\prime}, T^{\prime}\right)$, is evidently less strong.

\section{Comparison to two-phase coexistence in other processings}

Our interpretation of two-phase coexistence in as-milled $\mathrm{Fe}-\mathrm{Ni}$ involves a wider distribution of the coarse-grained free energy for the bcc phase than the fcc phase. This interpretation is based on the bcc phase accumulating a higher density of small, defective grains during milling. This type of enthalpy buildup may be unique to ball milling, so it is interesting to compare the composition range for two-phase coexistence in ball milled $\mathrm{Fe}-\mathrm{Ni}$ alloys to the composition range for two-phase coexistence for other nonequilibrium processings. Figure 10 compares the phase boundaries of the bcc and fcc phases of $\mathrm{Fe}-\mathrm{Ni}$ for five different types of processing. Thermodynamic equilibrium at room temperature shows a broad two-phase region, bounded on the right by $L 1_{2}$ ordered $\mathrm{Ni}_{3} \mathrm{Fe}$, which is a sluggishly ordering superstructure based on an fcc lattice. Thermal evaporation ${ }^{80}$ and sputtering $^{81}$ produce alloys with a narrower range of twophase coexistence, as expected. Note, however, that thermal evaporation and sputtering produce two-phase regions that are located more centrally in the two-phase region of the equilibrium diagram. In comparison, ball milling produces a two-phase region that is shifted to low Ni concentrations, and becomes more so with increased milling intensity. It seems that ball milling differs from the thin film preparation methods in that it produces a higher defect enthalpy in the bcc phase than the fcc phase. It would be interesting to compare the phase fractions across the two-phase regions for $\mathrm{Fe}-\mathrm{Ni}$ alloys synthesized by thermal evaporation or sputtering. 


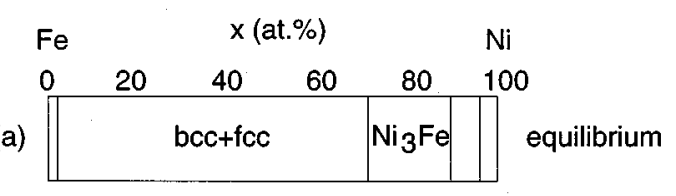

(b)

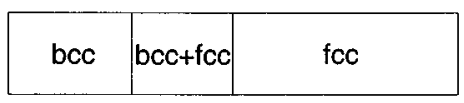

thermal evaporation

(c)

\begin{tabular}{|c|c|c|}
\hline bcc & $\begin{array}{c}\mathrm{bcc} \\
+ \\
\mathrm{fcc}\end{array}$ & fcc \\
\hline
\end{tabular}

sputtering

(d)

\begin{tabular}{|l|l|}
\hline $\mathrm{bcc}$ & \\
$\mathrm{bcc}$ & + \\
$\mathrm{fcc}$ & $\mathrm{fcc}$ \\
\hline
\end{tabular}

BM, lower intensity

(e)

\begin{tabular}{|c|c|}
\hline $\begin{array}{c}\mathrm{bcc} \\
\mathbf{b c c} \\
\mathbf{f c c}\end{array}$ & fcc \\
\hline
\end{tabular}

BM, higher intensity

FIG. 10. Phase boundaries for $\mathrm{Fe}-\mathrm{Ni}$ alloys processed by different methods: equilibrium (Ref. 73), evaporation (Ref. 80), sputtering (Ref. 81), ball milling at intensity $I_{0.3}$, and ball milling at intensity $I_{1}$.

\section{CONCLUSION}

We synthesized mechanically alloyed $\mathrm{Fe}_{100-x} \mathrm{Ni}_{x}$ alloys over the range of $x=0-49$. Our Spex mill was modified so that it could also operate at a significantly reduced milling intensity, and we compared the materials milled for long milling times with the normal and reduced intensities. We found that the composition ranges of the bcc and fcc singlephase regions were greatly extended with respect to their equilibrium ranges. At the higher milling intensity, we found that the bcc phase was destabilized with respect to the fcc phase, and the two-phase region shifted to lower Ni concentrations. Lattice parameters measured by x-ray diffractometry, and hyperfine magnetic fields measured by Mössbauer spectrometry, indicated that the chemical compositions of the individual phases in the as-milled alloys were approximately the same as the overall composition of the alloy. We therefore expect the thermodynamic phase preference of as-milled $\mathrm{Fe}-\mathrm{Ni}$ to be influenced by the polymorphic transformation line. In fact, the composition range for two-phase coexistence in as-milled $\mathrm{Fe}-\mathrm{Ni}$ does enclose the polymorphic transformation composition.

In DSC scans, we measured enthalpies for defect recovery in the fcc and bcc alloys of about 0.5 and $1.5 \mathrm{~kJ} / \mathrm{mol}$, respectively. X-ray line shape analysis showed that there was a large distribution in the internal strains of the as-milled crystallites. We found evidence for a distribution in the crystallite sizes, and a distribution in crystallite sizes in as-milled materials is generally expected. We propose that heterogeneities in the defect enthalpy of the fcc and (especially) the bcc phase will broaden the effective free-energy curves. We propose that local heterogeneities in the defect enthalpies are responsible for the region of two-phase coexistence around the polymorphic transformation composition. Near the polymorphic transformation composition, the free-energy curves of the bcc and fcc phase are not strongly separated, so it may be possible for highly defective bcc-rich regions to transform into fcc phase, even when the Ni concentration is a bit below the polymorphic transformation composition. We can predict semiquantitatively the composition range of two-phase coexistence by broadening upwards the Thermo-Calc free-energy curves by average amounts equal to the enthalpies measured in DSC scans for bcc and fcc alloys. The asymmetrical phase fraction curves can be understood by the differences in curvature of the bcc and fcc free-energy curves, and by a high energy tail of the bcc free-energy distribution. If the bcc free-energy distribution broadens upwards with milling intensity more than does the fcc free-energy distribution, we can understand the shift with milling intensity of the composition range for two-phase coexistence.

\section{ACKNOWLEDGMENTS}

We thank Professor John Morral and Dr. Hong Du for performing the Thermo-Calc calculations. This work was supported by the NSF under contract DMR-9213447.

${ }^{1}$ J. S. Benjamin, Mater. Sci. Forum 88-90, 1 (1992).

${ }^{2}$ C. C. Koch, O. B. Cavin, C. G. McKamey, and J. O. Scarbrough, Appl. Phys. Lett. 43, 1017 (1983).

${ }^{3}$ R. B. Schwarz, R. R. Petrich, and C. K. Saw, J. Non-Cryst. Solids 76, 281 (1985).

${ }^{4}$ E. Hellstern and L. Schultz, Appl. Phys. Lett. 48, 124 (1986).

${ }^{5}$ R. B. Schwarz, Mater. Sci. Eng. 97, 71 (1988).

${ }^{6}$ K. Suzuki, J. Non-Cryst. Sol. 112, 23 (1989).

${ }^{7}$ L. Schultz, Philos. Mag. B 61, 453 (1990).

${ }^{8}$ A. R. Yavari and P. Desré, Mater. Sci. Eng. A 134, 1315 (1991).

${ }^{9}$ M. A. Morris and D. G. Morris, J. Mater. Sci. 26, 4687 (1991).

${ }^{10}$ Y. Chen, M. Bibole, R. Le Hazif, and G. Martin, Phys. Rev. B 48, 14 (1993).

${ }^{11}$ Y. Chakk, S. Berger, B. Z. Weiss, and E. Brook-Levinson, Acta Metall. Mater. 42, 3679 (1994).

${ }^{12}$ M. Abdellaoui and E. Gaffet, J. All. Comp. 209, 351 (1994).

${ }^{13}$ E. Hellstern, H. J. Fecht, Z. Fu, and W. L. Johnson, J. Mater. Res. 4, 1292 (1989).

${ }^{14}$ H. J. Fecht, E. Hellstern, Z. Fu, and W. L. Johnson, Adv. Powder Metall. 1-2, 111 (1989).

${ }^{15}$ B. Fultz, G. LeCaër, and P. Matteazzi, J. Mater. Res. 4, 1450 (1989).

${ }^{16}$ H. J. Fecht, E. Hellstern, Z. Fu, and W. L. Johnson, Metall. Trans. A 21, 2333 (1990).

${ }^{17}$ E. Hellstern, H. J. Fecht, Z. Fu, and W. L. Johnson, J. Appl. Phys. 65, 305 (1989).

${ }^{18}$ J. S. C. Jang and C. C. Koch, J. Mater. Res. 5, 325 (1990).

${ }^{19}$ H. Bakker, L. M. Di, and D. M. R. Lo Cascio, Solid State Phenomena 23-24, 253 (1992).

${ }^{20}$ H. Bakker, G. F. Zhou, and H. Yang, Prog. Mater Sci. 39, 159 (1995).

${ }^{21}$ R. W. Cahn, in Diffusion in Ordered Alloys, edited by B. Fultz, R. W. Cahn, and D. Gupta (TMS, Warrendale, 1993), p. 125.

${ }^{22}$ H. Schröpf, C. Kuhrt, E. Arzt, and L. Schultz, Scr. Metall. Mater. 30, 1569 (1994).

${ }^{23} \mathrm{~S}$. Gialanella, Intermetallics (to be published).

${ }^{24}$ S. Begin-Colin, G. Le Caër, A. Mocellin, and M. Zandona, Philos. Mag. Lett. 69, 1 (1994).

${ }^{25}$ S. Begin-Colin, G. Le Caër, M. Zandona, E. Bouzy and B. Malaman, J. All. Comp. 227, 157 (1995).

${ }^{26}$ C. C. Koch and M. S. Kim, J. de Physique C 8, 573 (1985).

${ }^{27}$ E. Hellstern, L. Schultz, R. Bormann, and D. Lee, Appl. Phys. Lett. 53, 1399 (1988)

${ }^{28}$ C. C. Koch, Annu. Rev. Mater. Sci. 19, 121 (1989).

${ }^{29}$ A. Calka and A. P. Radlinski, Scr. Metall. 23, 1497 (1989).

${ }^{30}$ C. Suryanarayana and F. Froes, J. Mater. Res. 5, 1880 (1990).

${ }^{31}$ C. Suryanarayana and R. Sundaresan, Mater. Sci. Eng. A 131, 237 (1991).

${ }^{32}$ K. Uenishi, K. F. Kobayashi, K. N. Ishihara, and P. H. Shingu, Mater. Sci. Eng. A 134, 1342 (1991).

${ }^{33}$ M. V. Zdujic, K. F. Kobayashi, and P. H. Shingu, J. Mater. Sci. 26, 5502 (1991). 
${ }^{34}$ D. K. Mukhopadhyay, C. Suryanarayana, and F. H. Froes, Scr. Metall. Mater. 30, 133 (1994).

${ }^{35}$ G. Veltl, B. Scholz, and H.-D. Kunze, Mater. Sci. Eng. A 134, 1410 (1991).

${ }^{36}$ K. Sakurai, Y. Yamada, C. H. Lee, T. Fukunaga, and U. Mizatuni, Mater. Sci. Eng. A 134, 1414 (1991).

${ }^{37}$ C. Bansal, Z. Q. Gao, L. B. Hong, and B. Fultz, J. Appl. Phys. 76, 5961 (1994).

${ }^{38}$ J. Eckert, J. C. Holzer, and W. L. Johnson, J. Appl. Phys. 73, 131 (1993).

${ }^{39}$ J. Eckert, J. C. Holzer, C. E. Krill III, and W. L. Johnson, J. Appl. Phys. 73, 2794 (1993).

${ }^{40}$ J. Eckert, J. C. Holzer, C. E. Krill III, and W. L. Johnson, J. Mater. Res. 7, 1980 (1992).

${ }^{41}$ E. Ma, F. Brunner, and M. Atzmon, J. Phase Equil. 14, 137 (1993).

${ }^{42}$ E. Ma, M. Atzmon, and F. Pinkerton, J. Appl. Phys. 74, 995 (1993).

${ }^{43}$ A. R. Yavari, Phys. Rev. Lett. 70, 3522 (1993).

${ }^{44}$ E. Ma and M. Atzmon, Mater. Chem. Phys. 39, 249 (1995).

${ }^{45}$ A. W. Weeber, P. I. Loeff, and H. Bakker, J. Less-Common Metals 145, 293 (1988).

${ }^{46}$ T. J. Tiainen and R. B. Schwarz, J. Less-Common Metals 140, 99 (1988).

${ }^{47}$ M. Oehring, T. Klassen, and R. Bormann, J. Mater. Res. 8, 2819 (1993).

${ }^{48}$ E. Gaffet, M. Harmelin, and F. Faudot, J. Alloys Compounds 194, 23 (1993).

${ }^{49}$ L. B. Hong, C. Bansal, and B. Fultz, Nanostruct. Mater. 4, 949 (1994).

${ }^{50}$ Y. Abe and W. L. Johnson, J. Jpn. Soc. Powder Powder Metall. 40, 272 (1993).

${ }^{51}$ Z. Fu and W. L. Johnson, Nanostruct. Mater. 3, 175 (1993).

${ }^{52}$ K. Yamada and C. C. Koch, J. Mater. Res. 8, 1317 (1993).

${ }^{53}$ E. Gaffet, Mater. Sci. Eng. A 132, 181 (1991).

${ }^{54}$ E. Gaffet, Mater. Sci. Eng. A 135, 291 (1991).

${ }^{55}$ J. Eckert, L. Schultz, and E. Hellstern, J. Appl. Phys. 64, 3224 (1988).

${ }^{56}$ Y. Chen, M. Bibole, R. Le Hazif, and G. Martin, Phys. Rev. B 48, 14 (1993).

${ }^{57}$ C. Kuhrt and L. Schultz, J. Appl. Phys. 73, 1975 (1993).

${ }^{58}$ L. B. Hong, L. Anthony, and B. Fultz, J. Mater. Res. 10, 126 (1995).

${ }^{59}$ L. B. Hong and B. Fultz, Phys. Rev. B 52, 6230 (1995).

${ }^{60}$ D. Basset, P. Matteazzi, and F. Miani, Mater. Sci. Eng. A 174, 71 (1994); 168, 149 (1993).
${ }^{61}$ L. H. Schwartz and J. B. Cohen, Diffraction from Materials (Springer, Berlin, 1987), p. 224.

${ }^{62}$ Z. Q. Gao, Ph.D. dissertation, California Institute of Technology, 1994.

${ }^{63}$ G. K. Williamson and W. H. Hall, Acta Metall. 1, 22 (1953).

${ }^{64}$ H. P. Klug and L. E. Alexander, X-Ray Diffraction Procedures (WileyInterscience, New York, 1974), p. 664.

${ }^{65}$ P. Scherrer, Gött. Nachr. 2, 98 (1918).

${ }^{66}$ H. P. Klug and L. E. Alexander, X-Ray Diffraction Procedures (WileyInterscience, New York, 1974), p. 656.

${ }^{67}$ H. Ouyang, B. Fultz, and H. Kuwano, in Nanophases and Nanocrystalline Structures, edited by R. D. Shull and J. M. Sanchez (TMS, Warrendale, 1994), p. 95.

${ }^{68}$ B. Fultz, H. Kuwano, and H. Ouyang, J. Appl. Phys. 76, 5691 (1994).

${ }^{69}$ J. B. Nelson and D. P. Riley, Proc. Phys. Soc. London 57, 160 (1945).

${ }^{70}$ H. P. Klug and L. E. Alexander, X-Ray Diffraction Procedures (WileyInterscience, New York, 1974), p. 594.

${ }^{71}$ Brent Fultz, in Mössbauer Spectroscopy Applied to Magnetism and Materials Science, edited by G. J. Long and Fernande Grandjean (Plenum, New York, 1993), Vol. 1, Chap. 1.

${ }^{72}$ B. Fultz and J. W. Morris, Jr., Phys. Rev. B 34, 4480 (1986).

${ }^{73}$ Editors T. B. Massalski, H. Okamoto, P. R. Subramanian, and L. Kacprzak, Binary Alloy Phase Diagrams, 2nd ed. (ASM International, 1990) Vol. 2, p. 1736.

${ }^{74}$ Joint Committee on Powder Diffraction Standards (The International Center for Diffraction Data, 1993) files 23-297 and 18-877.

${ }^{75}$ R. P. Reed and R. E. Schramm, J. Appl. Phys. 40, 3453 (1969).

${ }^{76}$ B. Sundman, B. Jansson, and J.-O. Andersson, Calphad 9(2), 153 (1985).

${ }^{77}$ Z. S. Xing, D. D. Gohil, A. T. Dinsdale, and T. G. Chart, National Physics Laboratory, DMA (A) 103, London, 1985.

${ }^{78}$ Y.-Y. Chuang, Y. A. Chang, R. Schmid, and J.-C. Lin, Metall. Trans. A 17, 1361 (1986).

${ }^{79}$ C. N. J. Wagner and M. S. Boldrick, Mater. Sci. Eng. A 133, 26 (1991).

${ }^{80}$ G. Dumpich, E. F. Wassermann, V. Manns, W. Keune, S. Murayama, and Y. Miyako, J. Magn. Magn. Mater. 67, 55 (1987).

${ }^{81}$ K. Sumiyama, M. Kadono, and Y. Nakamura, Trans. Jpn. Inst. Met. 24(4), 190 (1983). 